\title{
ANÁlise E CLASSIFICAÇÃO DAS QUESTÕES DAS DEZ PRIMEIRAS OLIMPÍADAS BRASILEIRAS DE ASTRONOMIA E ASTRONÁUTICA ${ }^{+*}$
}

\author{
Julio Daniel Blanco Zárate \\ Administración Nacional de Enseñanza Publica \\ Consejo de Enseñanza Secundaria \\ Montevideo - Uruguay \\ João Batista Garcia Canalle \\ Instituto de Física - UERJ \\ José Mateus Nobre da Silva \\ Bolsista de Iniciação Científica - Instituto de Física - UERJ \\ Rio de Janeiro - RJ
}

\section{Resumo}

Neste trabalho, apresentamos uma análise sobre os tipos de questões das dez primeiras provas das Olimpiadas Brasileiras de Astronomia e Astronáutica (OBA) e sobre a formulação das questões de resolução única nas provas de Astronomia, tentando apontar elementos para a elaboração de perguntas baseadas na racionalização das respostas, ou seja, na vinculação dos conhecimentos e não na simples colocação de dados a serem substituídos em equações previamente memorizadas. As questões foram classificadas segundo critérios para problemas fechados ou de resposta única e em relação às competências básicas necessárias utilizadas pelos alunos para resolver a questão. Segundo a forma de obter o resultado correto, as questões foram categorizadas como: conhecimen-

\footnotetext{
+ Analysis and classification of the first ten tests of the Brazilian Olympics of Astronomy and Astronautics

* Recebido: novembro de 2008. Aceito: julho de 2009.
} 
to direto (tipo 1), cálculo (tipo 2), raciocínio e conhecimento (tipo 3), compreensão (tipo 4) e questões de ciência, tecnologia e sociedade (tipo 5). Observa-se através das edições da OBA que os tipos de questões tendem a aparecer em todos os niveis de provas. Em cada um dos niveis, as questões do tipo 3 e do tipo 4 sustentam-se percentualmente no tempo, em contrapartida, observa-se uma redução percentual nas questões do tipo 1. O número de questões que usam a simples memorização de respostas no Ensino Médio diminuiu ao longo do tempo também. Por exemplo, na I OBA havia $32,4 \%$ e na II OBA havia $53,3 \%$ de questões de simples memorização, mas, na prova da IX OBA, a percentagem foi de $10 \%$, havendo ainda uma redução de $2,9 \%$ da IX para a X OBA. As classificações e os resultados detalhados são apresentados, mostrando que as questões da OBA, onde possivel, têm convergido para mais questões do tipo 3 e do tipo 4 e menos para as do tipo 1. Nas provas das séries iniciais, a percentagem sobre as questões de simples memorização, ou seja, do tipo 1, passou de 77,8\% na VII OBA e para $35,7 \%$ na X OBA. A maior diferença de percentagem ocorreu nas provas da $5^{a}$ à $8^{a}$ séries (regime de 8 anos) ou da $6^{a}$ à $9^{a}$ séries (regime de 9 anos) do Ensino Fundamental, onde passou de 35,7\% para 5,6\%, para as mesmas VII e XOBA.

Palavras-chave: Olimpíada de Astronomia; formulação de questões; classificação; conhecimento declarado; conhecimento procedimental.

\begin{abstract}
In this work we present an analysis on the types of question from the first ten tests of the Brazilian Olympics of Astronomy and Astronautics (OBA). This paper examines the formulation of unique answer questions in the Astronomy issues, trying to point out elements for the elaboration of questions based on the rationalization of the answers, i.e. the linking of knowledge and not the simple placement of data to be substituted in previously memorized equations. The questions were classified according to closed problems or unique answer questions criteria, which are the basic skills necessary for students to use the time to resolve them. The
\end{abstract}


ways to get the right result, the questions were classified as: direct knowledge (type 1), calculation (type 2), reasoning and knowledge (type 3), understanding (type 4) and questions of Science technology and society (type 5). It is observed through the editions of OBA that the types of questions tend to appear in all level tests. In each level the type 3 and type 4 questions, claims the same percentage, however, there is a percentage reduction on type 1. The number of questions that use the simple memorization of responses in High School has decreased over time as well. For example, in the I OBA questions of simple memorization there was a $32.4 \%$ and $53.3 \%$ in II OBA, but in the test of the IX $O B A$ the percentage was $10 \%$ and there is still a reduction of $2.9 \%$ from the $I X$ to the X OBA. The classification and the detailed of the results are presented showing that the questions of $O B A$, wherever possible, have converged to more questions of the 3 and 4 types and less on questions of type 1. In the initial series of tests, the percentage of simple memorization questions, or type 1 decreased from $77.8 \%$ in VII OBA to $35.7 \%$ in the $X O B A$. The largest difference in percentage occurred in the 5th to 8th series (eight years scheme) or 6th to 9th series (9 years scheme) of Elementary School, where the percentage reduced from $35.7 \%$ to $5.6 \%$, since the VII to the XOBA.

Keywords: Astronomy Olympics; question formulation; classification; declared knowledge; procedure knowledge.

\section{Introdução}

A análise das perguntas foi feita segundo as características do tipo de conhecimento ou competência exigida do aluno no momento de achar a resposta certa.

A investigação destaca que na solução de problemas entram em jogo vários tipos de conhecimento (ERICKSON; SMITH, 1991; POZO, 1994). Esses conhecimentos tendem a ser mais específicos quanto mais fechados são os problemas para uma disciplina. Não é fácil ensinar ao aluno "a pensar em problemas", e é 
muito mais difícil ensiná-lo a "resolver problemas" (BRANDSFORD, 1989; HALPERN, 1992).

O domínio de técnicas e estratégias por parte do aluno (ou qualquer indivíduo), não é uma competência que se desenvolva independentemente dos conteúdos (CHINN; BREWER, 1993; CARRETERO, 2005). Além do que, todo conhecimento deve estar contextualizado para ser efetivo. As ideias, técnicas e estratégias para solução de problemas se desenvolvem a partir de conhecimentos e informações, que são (DRIVER, 1988):

a) específicas do domínio e dependem da tarefa usada para identificá-la;

b) difíceis de identificar, pois formam parte do conhecimento implícito do indivíduo;

c) pessoais porque, além das semelhanças entre as representações dos sujeitos, a interpretação depende do contexto individual.

Muitas dessas ideias também são guiadas pela percepção e experiência do indivíduo e em cada um deles nem sempre tem a mesma eficácia, nível de especificidade e potencial de generalização.

Mesmo tendo autores como Disessa e Solomon (1983), que pensam que o conhecimento infantil é desintegrado e fragmentário, não deveríamos acreditar sempre nisso, pois como apontam Vosniadou e Brewer (1992), o conhecimento é altamente dependente das experiências, e as crianças podem integrar esses conhecimentos ao contexto diário, assim como as experiências procedentes dos adultos em modelos estratégicos que usam de forma consciente.

As pesquisas mostram que os processos de raciocínio não independem do conteúdo, pelo contrário, são desenvolvidos a partir destes. As habilidades, competências, técnicas e estratégias são desenvolvidas no contexto de aplicação. Quanto mais competências são exigidas de um indivíduo, mais oportunidades de colocá-las em uso ele terá, e assim haverá maiores oportunidades de desenvolvimento de estratégias, competências e habilidades.

A ideia central é que cada professor elabore as técnicas de acordo não somente com os conteúdos que ele vai avaliar, mas também quais são as competências, habilidades e estratégias que ele quer que os alunos desenvolvam. Se um questionário, uma prova ou qualquer outra ferramenta de avaliação pretende somente medir os conhecimentos declarativos ou simplesmente o nível de informação do aluno, o professor está perdendo a oportunidade de contemplar o desenvolvimento de novas competências do indivíduo.

Desse jeito, o resultado da avaliação é só um algoritmo que mede a quantidade de informação certa (ou concordante com a ciência normal) que tem um 
aluno. Mas quando o professor gera situações em que o aluno é exigido a pensar, aplicar o conhecimento em forma não algorítmica, quando o professor contextualiza e coloca em jogo outras competências e capacidades dos alunos, ele está criando oportunidades para o desenvolvimento das habilidades exigidas.

Essas habilidades, competências e estratégias podem ser medidas quando o aluno é colocado em situações nas quais deve resolver uma questão. Usando-as nesse sentido, o conhecimento medido não é simplesmente o conhecimento declarativo, não se trata somente de saber "o que", mas também "o como" (ANDERSON, 1983). Desse jeito, os conhecimentos declarativos e as informações tradicionais adquiriram características próprias como produto da aprendizagem.

\section{Metodologia e resultados}

As questões da OBA foram classificadas em cinco tipos segundo destrezas, capacidades e competências exigidas dos alunos. Todas as questões tinham respostas únicas, ou seja, eram problemas fechados. Isto é necessário, pois de outro modo, com a inclusão de questões abertas, a correção seria inviável, porque os professores representantes da olimpíada em cada escola são os responsáveis pela correção delas por meio de um gabarito que é enviado a eles.

A partir dos critérios demarcatórios, as questões foram classificadas como:

\section{II.1 Questões de conhecimento direto: Tipo 1}

São aquelas nas quais o aluno deve ter um conhecimento específico para responder, cujo enunciado é simples. Podem ser questões de múltiplas opções ou de enunciado direto. Por exemplo:

Quais são os planetas que têm sistema de anéis? (Q1(a) / Nível 1 da I OBA).

Qual planeta tem o eixo de rotação menos inclinado com relação à perpendicular ao plano de sua órbita? (Q2(g) / Nível 2 da II OBA).

Nesse caso, a questão solicita somente que o aluno declare o conhecimento. Nas fases iniciais, parte do conhecimento deve ser adquirida com o uso da memória para guardar a informação e a outra parte desse mesmo conhecimento deve estar baseada na compreensão, no raciocínio da informação e seus devidos usos. 
Assim sendo, parte do conhecimento deve ser aprendido por técnicas de memória e a outra parte deve ser assimilada via raciocínio.

Essas técnicas devem ser mais trabalhadas quanto menos informações o aluno tiver, pois as técnicas e competências que derivam das aplicações de estratégias só podem ser aprendidas após o aluno possuir certas informações. Uma estratégia só pode ser desenvolvida sob contexto, e é necessário conhecer a informação para sua aplicação.

Um aluno não deve fazer uma pesquisa sempre que for colocado à frente de um novo problema. Ele deve ter aprendido os procedimentos e as informações de modo a usá-los de forma automática sempre que necessário, ou seja, quase inconscientemente. Trata-se de dominar certas destrezas com a finalidade de liberar os recursos cognitivos para enfrentar outras tarefas relacionadas com a estratégia da resolução do problema.

Encontrou-se um grande número de questões do tipo 1 nas provas da OBA do Ensino Fundamental, mas também foi encontrado no Ensino Médio, contudo, com percentagens decrescente no tempo. O número de questões do tipo 1 nas provas de nível 4 diminuiu de $32,4 \%$ (I OBA) e $53,2 \%$ (II OBA) para 10,0\% e $7,1 \%$ nas provas da IX e X OBA, até a V OBA o nível 1 era destinado a $1^{\mathrm{a}}$ até $4^{\mathrm{a}}$ séries do Ensino Fundamental (Fig. 1).

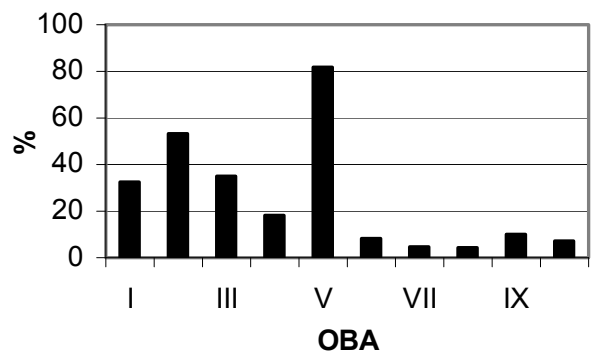

Fig. 1 - Observa-se a redução das questões do tipo 1 no nível 4 ao decorrer das dez edições da $O B A$.

Contudo, nas provas das séries inicias (nível 1), a percentagem passou de $77,8 \%$ para $35,7 \%$ da VII OBA para a X OBA (Fig. 2). Na prova do nível 1 da II OBA a tendência de colocar questões do tipo 1 era majoritária, atingindo $57,1 \%$ das questões analisadas; já na X OBA houve uma maior distribuição no tipo de questões. Mesmo sendo as questões do tipo 1 a maioria, acham-se agora questões de tipo 3, 4 e 5 em percentagens superiores a 14\%. A maior diferença de percenta- 
gem está nas provas do nível 3, que passou de 47,6\% da I OBA para 5,6\% na X OBA (Fig. 3), mesmo quando em várias oportunidades a percentagem de questões com uso de informação concreta ficou acima de $60 \%$ como nas IV e VI OBA.

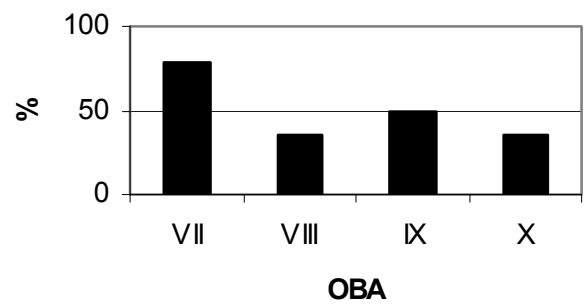

Fig. 2 - A partir da VI OBA, na qual começam as provas de Nivel 1 (contendo somente $1^{a}$ e $2^{a}$ séries do Ensino Fundamental), observa-se também que existe um declínio das percentagens ao longo dos anos. Esse declínio é muito mais suave, pois esse tipo de questão é necessário para as séries iniciais.

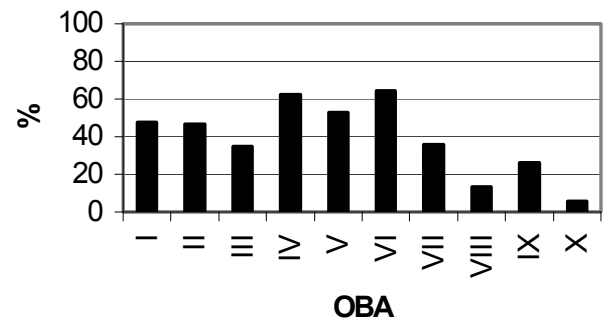

Fig. 3 - No nível 3, a redução é mais drástica após a VI OBA, onde os alunos devem se concentrar em questões de outros tipos, como as dos tipos 3 e 4 .

Vale a pena esclarecer que, às vezes, uma questão pode ser avaliada como sendo de memória para os alunos das séries finais e como de raciocínio para as séries iniciais. Isso se deve ao fato de que certos conhecimentos são inicialmente procedimentais ou explicativos e logo se transformam (ou deveriam se transformar) em conhecimentos declarativos após usos contínuos e sistêmicos (WELLINGTON, 1989). 


\section{2 Questões de cálculo: Tipo 2}

São questões nas quais o aluno deve ter conhecimento de um algoritmo ou equação para resolvê-las. Claro que não é somente necessário conhecer o algoritmo, o aluno deve saber aplicá-lo, substituir as variáveis e realizar as operações matemáticas necessárias. Essas questões variam muito em dificuldade, dependendo do nível da prova. Por exemplo:

O diâmetro do Sol é de, aproximadamente, $\boldsymbol{d}_{\text {Sol }}=1.400 .000 \mathrm{~km}$ e o diâmetro da Lua é de, aproximadamente, $\boldsymbol{d}_{\text {Lua }}=3.500 \mathrm{~km}$, contudo, os dois astros possuem o mesmo diâmetro angular no céu. A distância da Terra à Lua é de aproximadamente $\boldsymbol{D}_{\text {Lua }}=400.000 \mathrm{~km}$. Esperamos que você já tenha aprendido o capitulo de triângulos semelhantes na matemática. Usando as relações dos triângulos semelhantes, determine a distância da Terra ao Sol $\left(\boldsymbol{D}_{S o}\right)$. Para que a sua resposta fique mais próxima do valor correto, por favor, subtraia da sua resposta $10.000 .000 \mathrm{~km}$, uma vez que fizemos alguns arredondamentos nos números acima. (Q9 / nível 2 da IV OBA).

Quanto vale um ano-luz em quilômetros? (Q2(a) / nível 1 da I OBA).

As questões de cálculo podem ser aplicadas nas séries iniciais para estabelecer os níveis de relação entre as operações simples e as estratégias de passo único. Essas questões não estavam presentes nas provas dos níveis 1 e 2 analisadas.

No nível 3, a percentagem mais alta ocorreu na VIII OBA e a mais baixa na VI e IX OBA, na qual o uso do recurso foi nulo. No nível 4, a percentagem mais alta foi na VII OBA, com $66,7 \%$, enquanto as percentagens mais baixas foram nas II e III OBA, com 5,9 e 8,3\%, respectivamente.

\section{3 Questões de raciocínio e conhecimento: Tipo 3}

Para responder às questões do tipo 3 , é necessário ter algum conhecimento, mas essa não é condição suficiente; é o tipo de questão de conhecimento contextualizado. $\mathrm{O}$ aluno deve saber interpretar um conhecimento ou lei, refletir sobre o resultado de uma equação ou aplicar o conhecimento sobre uma situação diferente da simples aplicação do algoritmo. Por exemplo:

Nós aqui da OBA gostamos muito da constelação do Cruzeiro do Sul e quase sempre fazemos perguntas sobre ela. Em 2004 fizemos uma pergunta muito parecida com esta que faremos agora. No dia 20 de junho, às 19 horas, alguém que more próximo do equador terrestre vai ver o Cruzeiro do Sul na posição 1 e fazer o movimento abaixo esquematizado. A que horas, aproximadamente, o Cru- 
zeiro do Sul vai estar na posição 2, na mesma noite? (aparece uma figura com o Cruzeiro do Sul girado de 90). (Q6(a) / nível 3 da X OBA)

O lado da Lua que nunca vemos e que chamamos de lado escuro, afinal é sempre escuro mesmo ou não? Justifique a sua resposta. (Q10(b) / nível 2 da IV OBA)

Esse é o caso em que o aluno deve usar estratégias de seleção de algoritmos, procurar por informações no texto e apelar a conhecimentos declarados (informações e algoritmos) e/ou conhecimentos explicativos. A estratégia simples tem cinco passos típicos (POZO; POSTIGO, 1993):

1) Aquisição da informação

2) Interpretação da informação

3) Análise da informação

4) Realização de inferências

5) Organização e comunicação da informação.

Em quase todas as edições da OBA aparecem esses tipos de questões para todos os níveis. No nível 1 da IX OBA do ano 2006 não tinham questões classificadas neste item; na X OBA ocorreram $35,7 \%$ e nas outras encontra-se uma percentagem próxima aos $25 \%$.

No nível 2, salvo na IX OBA, na qual não se encontram esse tipo de questões e na VI OBA, a qual possui apenas $6,9 \%$ desse tipo de questão, as questões de raciocínio aparecem numa frequência próxima de $30 \%$, sendo que esse tipo de questão possui a distribuição mais homogênea em todos os níveis das provas pesquisadas.

Já no nível 3, o uso desse tipo de questão é bem variado. Não se encontra na VI OBA e chegou a 50\% em 2006 na IX OBA. No nível 4, a partir de 2004, esse tipo de questão ocupou cerca de $32 \%$ das questões, com uma variação de apenas $4 \%$.

As questões de raciocínio e conhecimento permitem a concretização do conhecimento explicativo, que é base da metacognição (ERICE; DUBINI, 2000). No Ensino Médio e Fundamental, usar esses tipos de questões (tipo 3) permite desenvolver as destrezas necessárias para passar certos mecanismos e procedimentos para a fase de conhecimento declarado e assim liberar recursos cognitivos necessários para incrementar estratégias e desenvolver as competências necessárias para programar soluções de problemas. 


\section{II.4 Questões de compreensão: Tipo 4}

Dado um texto, os alunos devem analisá-lo e tirar as respostas dele. Seguem os exemplos abaixo.

O Sol tem temperatura superficial de $6.000^{\circ} \mathrm{C}$ (graus Celsius) e tem cor amarela, como você sabe. As Plêiades (também chamadas de "Sete Irmãs"), por outro lado, são estrelas jovens, têm temperatura superficial de aproximadamente $20.000^{\circ} \mathrm{C}$, são muito maiores do que o Sol e são da cor azul esbranquiçado. Quantas vezes as Plêiades são mais quentes do que o Sol? Além das estrelas amarelas e das azuis esbranquiçadas, também existem as estrelas vermelhas, como Aldebaran (que quer dizer: "Aquela que segue as Plêiades"), na constelação do Touro e tem cerca de $3.000^{\circ} \mathrm{C}$ (graus Celsius). Leia toda a questão 2 e ligue a cor da estrela com a sua temperatura. (Q2(a-b)/ Nível 3 da X OBA)

Na famosa obra de fição de J. R. R. Tolkien, "O Senhor dos Anéis", transformada em uma recente trilogia cinematográfica, encontramos a seguinte citação, dita pelo nobre Aragorn: "Tive uma vida dura e longa; e as milhas que se estendem entre este lugar e Gondor são uma pequena fração na soma de minhas viagens. Atravessei muitas montanhas e muitos rios, e pisei em muitas planicies, chegando até mesmo às regiões distantes de Rhûn e Harad, onde as estrelas são estranhas [diferentes das que ele conhecia]". Pergunta: Baseado nessa citação de Aragorn, você acha que a Terra Média, o mundo onde se passa o livro, é plana ou esférica? Por que? (Q2/ nível 4 da X OBA).

Questões desse tipo apontam, na sua essência, ao fortalecimento dos três primeiros passos da estratégia detalhada por Pozo e Postigo (1994). Claro que o aluno pode ter decorado a resposta ou não precisar dos dados apresentados no texto para achar a resposta. Mas a finalidade desse tipo de questão não é que o aluno dê respostas memorizadas e sim que ele faça a descoberta das informações dentro do próprio texto. O texto deve conter os dados, e a pergunta não deve ser direta, o aluno precisará de raciocínio para achar as informações e selecionar aquelas necessárias para resolver a questão.

No nível 1, esse tipo de questão não é cabível, pois exigem longos textos e alunos desses níveis ainda estão sendo alfabetizados.

No nível 2, questões desse tipo só apareceram na VI OBA, com 10,3\% de frequência, alcançando um máximo na IX OBA com 17,6\% e um mínimo na VIII OBA com 7,2\% (Fig. 4). No nível 3, apareceram quase de forma contínua após a VI OBA, com uma percentagem de $21,4 \%$ e permanecendo com uma variância de aproximadamente $4 \%$ (Fig.5). 
No nível 4 da OBA, esse tipo de questão já apareceu na II OBA, numa única questão de caráter histórico, e o mesmo aconteceu na III OBA. Contudo, na IV OBA, já constituiu cerca de $23 \%$ das questões e alcançou valores de $39 \%$ na VII e X OBA. Nas VIII e IX OBA apresentou valores próximos a 20\% (Fig. 6).

As questões de compreensão incentivam a capacidade de selecionar informações. Como é que isso ajuda a planejar estratégias de resolução? Adquirindo a competência o indivíduo aprende a administrar os recursos cognitivos aprendendo a valorizar quais são as informações necessárias e quais são as informações que podem ser descartadas.

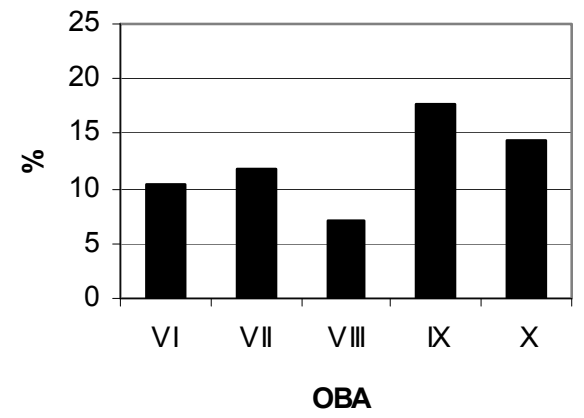

Fig. 4 - Para o nivel 2, questões do tipo 4 não são encontradas nas edições ocorridas da I OBA até a V OBA. Após esse periodo, acompanha-se um leve crescimento desse tipo de questão no decorrer dos anos.

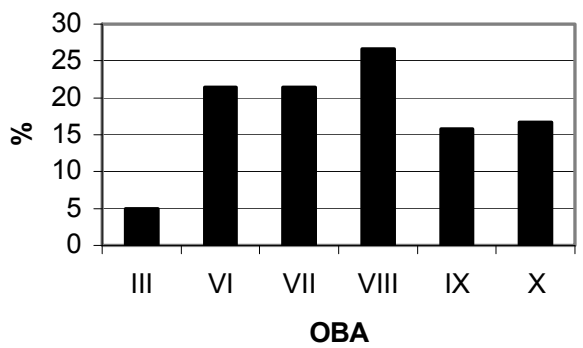

Fig. 5 - Para o nível 3, questões do tipo 4 aparecem com uma maior frequência após a V OBA. Para a I OBA e V OBA não foram encontradas questões do tipo 4. Os alunos desse nivel devem ser capazes de resolver essas questões. 


\section{II.5 Questões CTS: Tipo 5}

Comumente CTS são as iniciais de Ciência Tecnologia e Sociedade, por isso foram incluídas aqui aquelas questões nas quais os alunos devem dar uma opinião sobre um fato, sobre uma data histórica, dar opinião técnica ou política sobre um evento acontecido ou provável. Além de terem o conhecimento científico, devem aplicar critérios sociais ou estabelecer juízos de caráter social ou histórico. Nessas questões, os alunos podem fazer até algum tipo de cálculo, mas o mais importante não é o resultado do cálculo e sim os aspectos sociais, históricos ou culturais. Vejam os exemplos abaixo:

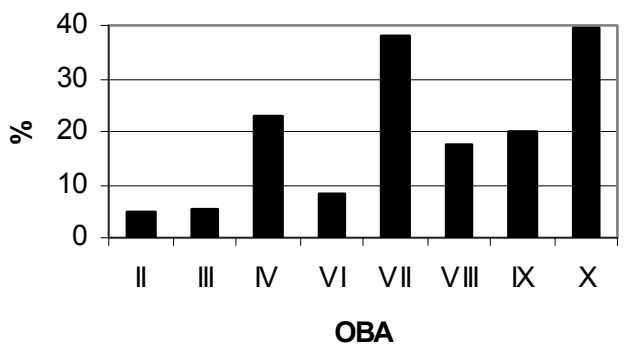

Fig. 6 - Esse gráfico demonstra o desenvolvimento das questões do tipo 4 no nível 4 ao longo das edições da OBA. Não foram encontradas questões desse tipo na I e VOBA.

As imagens da superficie da Terra, obtidas a partir de satélites, são muito úteis no estudo do tempo, de oceanos, rios, cidades, florestas e culturas agrícolas. Abaixo está a imagem da cidade de Belém, capital do estado do Pará; foi obtida pelo satélite CBERS, que foi construído por brasileiros e chineses. Abaixo da figura grande tem 4 figuras pequenas. Faça um grande $X$ sobre aquela que não pertence à figura grande (é apresentada uma figura grande e 4 figuras pequenas). (Q10(a) / Nível 1 da X OBA)

No Brasil existem cientistas que trabalham na construção de foguetes e satélites. Eles constroem satélites no Instituto Nacional de Pesquisas Espaciais (INPE) e foguetes no Instituto de Aeronáutica e Espaço (IAE), órgão do Comando-Geral de Tecnologia Aeroespacial (CTA). Para coordenar as atividades espaciais brasileiras existe a Agência Espacial Brasileira (AEB) que, por meio do Programa AEB Escola, promove atividades educacionais em escolas do Brasil. Neste ano de 2006, o Brasil comemora os 100 anos do voo do 14-Bis. Esse voo foi 
realizado pelo brasileiro Alberto Santos Dumont em 1906, na cidade de Paris, na França. Pinte, de qualquer cor, a figura que representa o 14-Bis (São dadas quatro figuras). (Q8 / Nível 2 da IX OBA)

Em quase todas as provas da OBA sempre se teve o cuidado de colocar questões relacionadas à história brasileira, história da Astronomia e questões ligadas à atualidade da tecnologia ou à atualidade política e cultural brasileira. Embora as percentagens ainda não sejam estáveis como no caso dos outros tipos de questões, pode-se afirmar que a mesma vem se instalando, principalmente após a inclusão da Astronáutica na OBA.

Esclarecemos, por fim, que em várias questões havia múltiplas perguntas e, nesse caso, cada pergunta era contada como uma questão.

\section{Considerações finais}

Com um percentual de até $40 \%$ nas provas das séries iniciais do nível 1 e 2, e uma redução na percentagem para menos de $10 \%$ nas provas do nível 3 e 4 , pode-se afirmar que há uma tendência de estabilização nas questões do tipo 1. Essa distribuição de questões que passa por todos os tipos (conhecimento direto, cálculo, raciocínio e conhecimento, compreensão, Ciência Tecnologia e Sociedade) parece ser a mais apropriada para o modelo de prova e os objetivos da OBA.

Observa-se, portanto, que os estilos das questões da OBA eram inicialmente predominantes nos tipos 1 e 3, mas têm variado no decorrer das diferentes OBA, criando-se uma gama variada de perguntas que apontam para diferentes intenções pedagógicas e didáticas. Todo tipo de questão é válida e necessária, pois elas vão requerer diferentes tipos de competências e habilidades que o aluno deve treinar para apreender.

As questões de cálculo aparecem somente nos níveis 3 e 4, permitindo avaliar também a relação integral entre conhecimento e uso direto. Essas questões deveriam continuar sendo evitadas nos níveis iniciais, porém elas são necessárias no nível 4.

As questões de raciocínio e conhecimento levam o aluno ao estágio de postulação de estratégias na aplicação de conhecimentos já adquiridos. Isso é importante em todos os níveis, pois gera a consciência de que os conhecimentos têm utilidade, além do que, cada estratégia aprendida é um novo tipo de conhecimento adquirido. 
As questões de compreensão permitem avaliar as possibilidades cognitivas do aluno numa determinada área. Elas também verificam a disponibilidade de recursos cognitivos próprios; o que o aluno é capaz de conhecer mesmo quando o objeto a ser conhecido não seja apresentado e explicado por outra pessoa. Permite, nesse caso, verificar as capacidades do novato em algum momento virar um experto ou especialista.

As questões sobre Ciência, Tecnologia e Sociedade iniciam o aluno na vida como cidadão, mesmo não virando especialista em ciência, logo ele compreenderá que existe uma relação muito forte entre o conhecimento, a história, a economia e o desenvolvimento de uma sociedade. Esses aspectos do conhecimento deveriam estar sempre incluídos nos níveis 3 e 4 , idade propícia para o desenvolvimento da consciência cívica.

Vale a pena esclarecer que logo após a IX OBA começaram a aparecer questões práticas que não foram avaliadas neste trabalho. Essas questões têm a ver com o desenvolvimento de conhecimento aplicado, assim como das destrezas manuais dos alunos.

As atividades práticas ou de criatividade ajudam a aproximação afetiva docente-aluno, o que constitui um importante fator de vínculo que ajuda ao estudante a se predispor a aprender o que o professor tenta ensinar.

As atividades práticas, bem orientadas e não dirigidas, também ajudam a desenvolver estratégias aplicadas e contextualizadas muito melhor que os problemas, inclusive os abertos. Elas permitem ao aluno refletir sobre fatos reais, redirecionar o conhecimento, reagir e reformular soluções possíveis, procurar alternativas, aceitar fracassos e assumir êxitos. Principalmente quando o professor sabe que o verdadeiro ator dessa peça deve ser o aluno.

\section{Referências bibliográficas}

ANDERSON, B. The experimental gestalt of causation: a common core to pupils' preconceptions in science. European Journal of Science Education, v. 8, n. 2, p. 155-171, abr. 1986.

BRANDSFORD, J. D.; FRANKS, J. J.; VYE, N. J.; SHERWOOD, R. D. New approaches to instruction: because wisdom can't be told. New York: Cambrige Univesity Press, 1989.

CARRETERO, M. et al. Construir y enseñar las ciencias experimentales. Buenos Aires: Aique Editora, 2005. 
CHINN, C. A.; BREWER, W. An empirical test of a taxonomy of responses to anomalous data in science. Journal of Research in Science Teaching, v. 35, n. 6, p. 623-654, ago. 1998.

DRIVER, R. Un enfoque constructivista para el desarrollo del currículo de ciencias. Enseñanza de las Ciências: revista de investigación y experiencias didácticas, v. 6, n. 2, p. 109-120, 1988.

DISESSA, A. Toward an epistemology of Physics. Cognition and Instruction. Journal Article, v. 10, p.105-225, 1993. Disponível em:

$<$ http://www.questia.com> Acesso em: 2 set. 2008.

ERICE, M. X.; DUBINI, L. Estrategias de resolución de problemas en el aprendizaje de contenidos de Física en el 3er ciclo de la EGB. Disponível em: http://www.fisica.ucr.ac.cr/varios/ponencias/5estrategias\%20de\%20resolucion.pdf

ERICKSON, K. A.; SMITH, J. Toward a theory of expertise. Cambridge: Cambridge Press, 1991.

HALPERN, D. F. Teaching and learning on the edge of the millennium: building on what we have learned: new directions for teaching and learning. Teaching for critical thinking: helping college students develop the skills and dispositions of a critical thinker. Hoboken, N. J.: WILEY Editora, 1999. p. 69-74.

MOORE, F.; MOORE, C. F.; WARD, H; WARD, D. Develop problem solving skills. Disponível em: $<$ https:/hw.serv.ph.utexas.edu/bur/solvingSkills.html $>$.

POZO, J.; GÓMEZ CRESPO, M. La solución de problemas. La solución de problemas en ciencias de la naturaleza. Madri: Santillana, 1994. p. 86-126.

POZO, J. I. Psicología y Didáctica de las Ciencias de la naturaleza: ¿concepciones alternativas? Infancia y Aprendizaje, n. 62-63, p. 187-204, 1993. Disponível em: $<$ http://dialnet.unirioja.es> Acesso em: 02 set. 2003.

POZO, J. I.; POSTIGO, I. Las estrategias de aprendizaje como contenido del currículo. In: MONEREO, C. (Comp.). Las estrategias de aprendizaje: Procesos, contenidos e interacción. Barcelona: Ediciones Doménech, 1993. 
SOLOMON, J. Science in a social context. Londres: Blackwell and the Association for Science Education, 1983.

VOSNIADOU, S.; BREWER, W. Mental models of the earth. Cognitive Psychology, n. 24, p. 535-585, 1992.

VOSNIADOU, S. Capturing and modelling the process of conceptual change. Learning and instruction, v. 4, n. 1, p. 45-69, 1994.

WELLINGTON, J. J. Practical work in School Science: which way now? Routledge Editora, 1989. 293 p.

WELLINGTON, J. Formal and informal learning in science: the role of the interactive science centers. Physics Education, v. 25, p. 247-252, 1990.

ERICE, M. X. et al. Estrategias de resolución de problemas en el aprendizaje de contenidos de Física en el 3er ciclo de la EGB. Index of ponencias, Universidad de Costa Rica, 2007. Disponível em:

$<$ http://www.fisica.ucr.ac.cr/varios/ponencias > Acesso em: 2 set. 2008. 